TSUKUBA J. MATH.

Vol. 9 No. 2 (1985). $299-316$

\title{
STABLE EQUIVALENCE BETWEEN UNIVERSAL COVERS OF TRIVIAL EXTENSION SELF-INJECTIVE ALGEBRAS
}

\author{
By
}

Takayoshi WAKAMATSU

\section{Introduction.}

Let $A$ be an indecomposable basic artin algebra and $T_{A}$ a basic tilting module with $B=\operatorname{End}\left(T_{A}\right)$. Let us denote by $R$ and $S$ the trivial extension selfinjective algebras $A \ltimes D A$ and $B \ltimes D B$, respectively. In the papers [24] and [22], H. Tachikawa and the author have proved that there is a stably equivalent functor $\mathcal{S}: \underline{\bmod }-R \rightarrow \underline{\bmod }-S$ and the restriction of $\mathcal{S}$ to the tilting torsion class $\mathscr{I}=\left\{X \in \bmod -A \mid \operatorname{Ext}_{A}^{1}(T, X)=0\right\} \quad$ coincides with that of the tilting functor $\operatorname{Hom}_{A}(T, ?)$.

D. Hughes and J. Waschbüsch [18] introduced the following doubly infinite matrix algebra :

$$
\hat{A}=\left(\begin{array}{lllll}
\ddots & \ddots & & & 0 \\
\dot{A_{n-1}} & \dot{M}_{n-1} & & & \\
& A_{n} & M_{n} & & \\
& & A_{n+1} & M_{n+1} \\
& & & \ddots & \ddots \\
& & & \ddots
\end{array}\right)
$$

in which matrices are assumed to have only finitely many entries different from zero, $A_{n}=A$ and $M_{n}=D A$ for all integers $n$, all the remaining entries are zero, and multiplication is induced from the canonical maps $A \otimes_{A} D A \simeq D A, D A \otimes_{A} A \simeq$ $D A$ and zero maps $D A \otimes_{A} D A \rightarrow 0$.

The identity maps $A_{n} \rightarrow A_{n+1}, M_{n} \rightarrow M_{n+1}$ induce an algebra isomorphism $\nu_{A}$ of $A$. The orbit space $\hat{A} / \nu_{A}$ is easily seen to be the trivial extension algebra $R$. Similarly, we can consider the orbit space $A /\left(\nu_{A}\right)^{n}$ as a self-injective algebra and it is denoted by $R_{n}$ for each $n=1,2, \cdots, \infty$. Notice that $R_{1}=R$ and $R_{\infty}=\hat{A}$.

The aim of this article is to prove the existence of a stably equivalent functor $S_{n}: \underline{\bmod }-R_{n} \rightarrow \underline{\bmod }-S_{n}$ for each $n$. Here $S_{n}$ is an orbit space $B /\left(\nu_{B}\right)^{n}$. The desired functor $\mathcal{S}_{n}$ will be defined by slightly modifying the definition of the functor $\mathcal{S}=\mathcal{S}_{1}$ in [24] and [22]. 
In order to relate the categories $\bmod -\hat{A}$ and $\bmod -R$, Hughes-Waschbüsch used the exact functor $\Phi: \bmod -\hat{A} \rightarrow \bmod -R$ which preserves the indecomposability and the composition length of a module and also almost split sequences and irreducible maps. Similarly to the functor $\Phi$, we can define the functors $\Phi_{n}$ : $\bmod -\hat{A} \rightarrow \bmod -R_{n}$ and $\Phi_{m, n}: \bmod -R_{m \cdot n} \rightarrow \bmod -R_{n}$. We shall show that the functors $\mathcal{S}_{1}=\mathcal{S}, \mathcal{S}_{2}, \mathcal{S}_{3}, \cdots, \mathcal{S}_{\infty}$ make the following diagrams commutative :

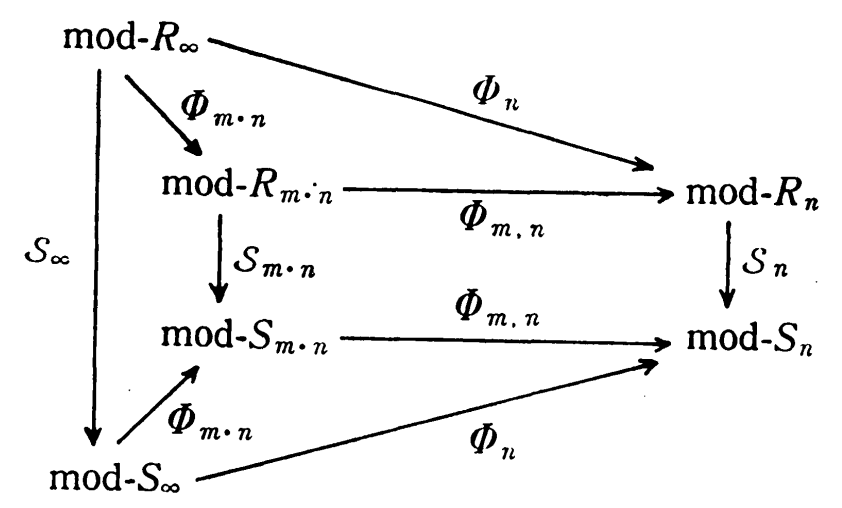

It should be noted that the functor $\Phi$ is not dense in general, though in the case where $R$ is representation-finite or $A$ is hereditary $\Phi=\Phi_{1}$ is dense and $\mathcal{S}=\mathcal{S}_{1}$ is induced from $\mathcal{S}_{\infty}$.

Recently, D. Happel [15] has proved that $\bmod -\hat{A}$ and $\bmod -\hat{B}$ are equivalent if gl. $\operatorname{dim} . A<\infty$. But, since $\Phi$ is not dense in general even if gl. dim. $A<\infty$, our results does not follow from his one. At the end of this paper such an example will be given.

Throughout this paper, we fix a commutative artin ring $K$ and all algebras are assumed to be artin $K$-algebras except $R_{\infty}$ and $S_{\infty}$, and modules are finitely generated over $K$ and morphisms operate on the opposite side of the scalars. The ordinary duality functor is always denoted by $D$.

\section{Preliminaries}

In this section, we shall recall some of basic results on tilting theory and trivial extension algebras for the later use.

Let $T_{A}$ be a tilting module in the sense of Happel-Ringel [16]. Put $B=$ $\operatorname{End}\left(T_{A}\right)$, then ${ }_{B} T$ is again a tilting module with $\operatorname{End}\left({ }_{B} T\right)=A$. Let us put $\mathscr{I}=$ $\left\{X \in \bmod -A \mid \operatorname{Ext}_{A}^{1}(T, X)=0\right\}, \mathscr{F}=\left\{X \in \bmod -A \mid \operatorname{Hom}_{A}(T, X)=0\right\}, \quad X=\{Y \in \bmod -B \mid$ $\left.Y \otimes_{B} T=0\right\}$ and $a f=\left\{Y \in \bmod -B \mid \operatorname{Tor}_{1}^{B}(Y, T)=0\right\}$. Further, let $F=\operatorname{Hom}_{A}(T$, ?), $F^{\prime}=\operatorname{Ext}_{A}^{1}\left(T\right.$, ?) (resp. $G=\left(\right.$ ? $\left.\otimes_{B} T\right), G^{\prime}=\operatorname{Tor}_{1}^{B}($ ?, $\left.T)\right)$ be functors from mod- $A$ (resp. $\bmod -B)$ to $\bmod -B(\operatorname{resp} . \bmod -A)$. Then there are short exact sequences of functors 


$$
\begin{aligned}
& 0 \longrightarrow G F \stackrel{\varepsilon}{\longrightarrow} 1_{\bmod \cdot A} \longrightarrow G^{\prime} F^{\prime} \longrightarrow 0, \\
& 0 \longrightarrow F^{\prime} G^{\prime} \longrightarrow 1_{\bmod \cdot B} \stackrel{\eta}{\longrightarrow} F G \longrightarrow 0,
\end{aligned}
$$

where $\varepsilon$ and $\eta$ denote the counit and the unit of the adjunction $(F, G)$, respectively. Hence the restrictions of the functors $F$ and $G$ (resp., $F^{\prime}$ and $G^{\prime}$ ) give a category equivalence $\mathscr{I} \cong \mathscr{G}$ (resp. $\mathscr{I} \cong \mathfrak{X}$ ).

We call a short exact sequence $0 \rightarrow X_{A} \rightarrow V_{A} \rightarrow L_{A} \rightarrow 0$ a torsion resolution of $X_{A}$ if $V \in \mathscr{T}$ and $L \in \operatorname{add}\left(T_{A}\right)$. There is the minimal torsion resolution $0 \rightarrow$ $X \stackrel{\alpha_{X}}{\longrightarrow} V(X) \stackrel{\beta_{X}}{\longrightarrow} T(X) \rightarrow 0$ for any $A$-module $X$ and every torsion resolution of $X$ is of the form

$$
0 \longrightarrow X \stackrel{\left(\begin{array}{c}
\alpha_{X} \\
0
\end{array}\right)}{\longrightarrow} V(X) \oplus T_{0} \stackrel{\left(\begin{array}{cc}
\beta_{X} & 0 \\
0 & 1_{T_{0}}
\end{array}\right)}{\longrightarrow} T(X) \oplus T_{0} \longrightarrow 0 .
$$

Similarly, a short exact sequence $0 \rightarrow W_{B} \rightarrow U_{B} \rightarrow Y_{B} \rightarrow 0$ is said to be a torsion-free resolution of $Y_{B}$ if $U \in Q$ and $W \in \operatorname{add}\left(D T_{B}\right)$. It is easy to see that the sequence $0 \rightarrow W_{B} \rightarrow U_{B} \rightarrow Y_{B} \rightarrow 0$ in the category mod- $B$ is a torsion-free resolution iff the corresponding sequence $0 \rightarrow_{B} D Y \rightarrow_{B} D U \rightarrow_{B} D W \rightarrow 0$ in the category $B$-mod is a torsion resolution. Therefore, there is the minimal torsion-free resolution $0 \rightarrow W(Y)$ $\stackrel{\delta_{Y}}{\longrightarrow} U(Y) \stackrel{\gamma_{Y}}{\longrightarrow} Y \rightarrow 0$ and every torsion-free resolution is of the form

$$
0 \longrightarrow W(Y) \oplus W_{0} \stackrel{\left(\begin{array}{cc}
\delta_{Y} & 0 \\
0 & 1_{W_{0}}
\end{array}\right)}{\longrightarrow} U(Y) \oplus W_{0} \stackrel{\left(\gamma_{Y}, 0\right)}{\longrightarrow} Y \longrightarrow 0 .
$$

Any module $X_{R}$ over the trivial extension self-injective algebra $R=A \ltimes D A$ is defined by giving its underlying $A$-module $X_{A}$ and the $A$-morphism $\phi: X \otimes_{A} D A$ $\rightarrow X$ such that $\phi \cdot(\phi \otimes D A)=0$ and any $R$-morphism $f: X_{R}=\left(X_{A}, \phi\right) \rightarrow X_{A}^{\prime}=\left(X_{A}^{\prime}, \phi^{\prime}\right)$ can be considered as an $A$-morphism $f: X_{A} \rightarrow X_{A}^{\prime}$ making the following diagram commutative :

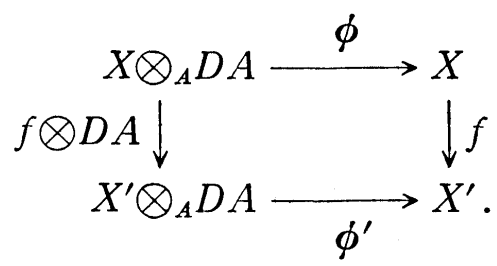

See [12] for details. If the underlying $A$-module $X_{A}$ is decomposed as a direct sum $X_{0} \oplus X_{1}$ and the morphism $\phi$ is of the form $\left(\begin{array}{cc}0 & 0 \\ \phi_{0} & 0\end{array}\right):\left(X_{0} \oplus X_{1}\right) \otimes D A \rightarrow\left(X_{0} \oplus X_{1}\right)$, we shall denote $X_{R}=\left(X_{A}, \phi\right)$ by $\underset{X_{1}}{X_{0}}$. It should be noted that any indecomposable projective (= injective) $R$-module has to be of the form $f R=\underset{f D A}{f A}$ with a primi- 
tive idempotent $f \in A \subset R$.

Similarly, by the definition, any object $X$ in the category mod- $R_{\infty}\left(R_{\infty}=\hat{A}\right)$ is is defined by giving a family of $A$-modules $\left\{X_{i}\right\}_{i \in \boldsymbol{Z}}\left(X_{i} \neq 0\right.$ for only finite number of integers $i \in \boldsymbol{Z})$ and a family of $A$-morphisms $\left\{\phi_{i}: X_{i} \otimes D A \rightarrow X_{i+1}\right\}_{i \in Z}$ satisfying $\phi_{i+1} \cdot\left(\phi_{i} \otimes D A\right)=0$ for all $i \in Z$. Any morphism in the category mod- $R_{\infty}$ from $X=\left\{X_{i}, \phi_{i}\right\}$ to $X^{\prime}=\left\{X_{i}^{\prime}, \phi_{i}^{\prime}\right\}$ is a family of $A$-morphisms $\left\{f_{i}: X_{i} \rightarrow X_{i}^{\prime}\right\}$ such that the following diagrams are commutative for all $i \in Z$ :

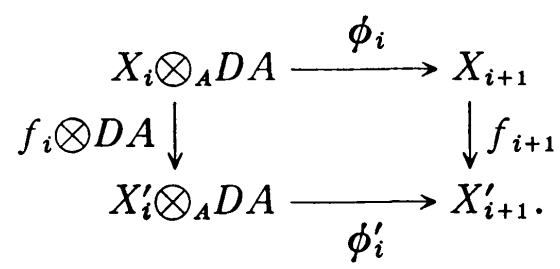

Similarly to the above also, for any positive integer $n$, an $R_{n}$-module $X$ is defined by giving a family of $A$-modules $X_{0}, X_{1} \cdots, X_{n-1}$ and a family of $A$ morphisms $\phi_{0}: X_{0} \otimes_{A} D A \rightarrow X_{1}, \cdots, \phi_{n-2}: X_{n-2} \otimes_{A} D A \rightarrow X_{n-1}$ and $\phi_{n-1}: X_{n-1} \otimes_{A} D A$ $\rightarrow X_{0}$ satisfying $\phi_{i+1} \cdot\left(\phi_{i} \otimes D A\right)=0$ for each $i=0,1, \cdots, n-1$. An $R_{n}$-morphism from $X=\left\{X_{i}, \phi_{i}\right\}$ to $X^{\prime}=\left\{X_{i}^{\prime}, \phi_{i}^{\prime}\right\}$ is a family of $A$-morphisms $f=\left\{f_{i}: X_{i} \rightarrow X_{i}^{\prime}\right\}$ such that $\phi_{i}^{\prime} \cdot f_{i} \otimes D A=f_{i+1} \cdot \phi_{i}$ for each $i$. Where we put $X_{i+s \cdot n}=X_{i}$ and $\phi_{i+8 \cdot n}=\phi_{i}$ $(1 \leqq i \leqq n, s \in N)$, for convenience.

Then the functors $\Phi_{n}: \bmod -R_{\infty} \rightarrow \bmod -R_{n}$ and $\Phi_{m, n}: \bmod -R_{m \cdot n} \rightarrow \bmod -R_{n}$ are defined as follows:

$$
\Phi_{n}\left(\left\{X_{j}, \phi_{j}\right\}\right)=\left\{Y_{i}, \phi_{i}\right\}_{i=1}^{n}, \quad Y_{i}=\underset{j \equiv i(\bmod n)}{X_{j}}
$$

and

$$
\begin{aligned}
& \phi_{i} \mid X_{j} \otimes D A=\phi_{j} \quad \text { for all } j \equiv i(\bmod n) . \\
& \Phi_{n}\left(\left\{f_{j}: X_{j} \rightarrow X_{j}^{\prime}\right\}\right)=\left\{g_{i}: \bigoplus_{j \equiv i} X_{j} \rightarrow \bigoplus X_{j}^{\prime}\right\}, \quad g_{i}=\bigoplus_{j \equiv i} f_{j} .
\end{aligned}
$$

It is easy to verify that the functors $\Phi_{n}, \Phi_{m, n}$ are exact and preserve the projectivity ( $=$ injectivity), indecomposability and composition length of a module and almost split sequences and irreducible maps. Further they make the following commutative diagrams :

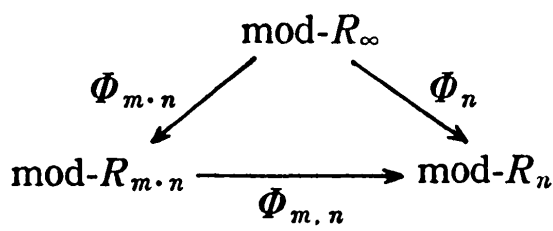

and

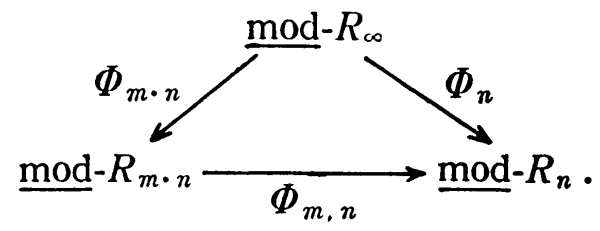

Here mod-* denotes the projectively (= injectively) stable category of mod-* in the sense of $M$. Auslander, for each self-injective algebra *. 
2. The functors $S_{n}: \underline{\bmod }-R_{n} \rightarrow \underline{\bmod }-S_{n}$ and $Q_{n}: \underline{\bmod }-S_{n} \rightarrow \underline{\bmod }-R_{n}$

In this section, we shall define the functor $\mathcal{S}_{n}: \underline{\bmod }-R_{n} \rightarrow \underline{\bmod }-S_{n}$ first and then, by making use of this functor $\mathcal{S}_{n}$, the functor $Q_{n}$ will be defined as the composite $\underline{\bmod }-S_{n} \stackrel{D}{\longrightarrow} S_{n}$ - $\underline{\bmod } \stackrel{\mathcal{S}_{n}}{\longrightarrow} R_{n} \underline{\bmod } \stackrel{D}{\longrightarrow} \underline{\bmod }-R_{n}$. Notice that, since $R_{n}$ and $S_{n}$ are self-injective, the duality functor $D: \bmod -R_{n} \rightleftarrows R_{n}$-mod, $\bmod -S_{n} \rightleftarrows S_{n}$-mod induces the duality functor $\underline{\bmod }-R_{n} \rightleftarrows R_{n}$ - $\underline{\bmod }, \underline{\bmod }-S_{n} \rightleftarrows S_{n}$ - $\underline{\bmod }$ (we denote this functor also by $D$ ). The functor $\mathcal{S}_{n}: S_{n}-\underline{\bmod } \rightarrow R_{n}$ - $\underline{\bmod }$ can be defined similarly to $S_{n}: \underline{\bmod }-R_{n} \rightarrow \underline{\bmod }-S_{n}$.

For an $R_{n}$-module $X=\left\{X_{i}, \phi_{i}\right\}$, we shall define $S_{n}$-modules $\mathcal{A}(X)$ and $\mathscr{B}(X)$ and $S_{n}$-monomorphism $u(X): \mathcal{A}(X) \rightarrow \mathscr{B}(X)$ and the module $\mathcal{S}_{n}(X)$ is defined as its cokernel Cok $u(X)$. In order to define those $S_{n}$-modules and $S_{n}$-morphism, the following lemma is necessary

LEMMA 2.1. ${ }_{A} D A_{A} \cong{ }_{A} D T \otimes_{B} T_{A}$ and ${ }_{B} D B_{B} \cong{ }_{B} T \otimes_{A} D T_{B}$.

Proof. Since ${ }_{B} T_{A}$ is a balanced bimodule, we have ${ }_{A} D A_{A} \cong{ }_{A} D \operatorname{Hom}\left({ }_{B} T,_{B} T\right)_{A}$ $\cong_{A} D T \otimes_{B} T_{A}$ and ${ }_{B} D B_{B} \cong{ }_{B} D \operatorname{Hom}\left(T_{A}, T_{A}\right)_{B} \cong{ }_{B} T \otimes_{A} D T_{B}$.

In the following, we can identify $D A$ (resp. $D B$ ) with $D T \otimes T$ (resp. $T \otimes D T$ ). Further, from the lemma, it follows that ${ }_{A} D A \otimes_{A} D T_{B} \cong{ }_{A} D T \otimes_{B} T_{A}$ and ${ }_{B} T \otimes_{A} D A_{A}$ $\cong_{B} D B \otimes_{B} T_{A}$ and we shall identify these bimodules respectively.

Now let us put $\mathcal{A}(X)=\left\{X_{i} \otimes D T,-\phi_{i} \otimes D T: X_{i} \otimes D T \otimes D B=X_{i} \otimes D A \otimes D T \rightarrow\right.$ $\left.X_{i+1} \otimes D T\right\}$ and $\mathcal{B}(X)=\left\{F\left(V\left(X_{i+1}\right)\right) \oplus F\left(V\left(X_{i}\right)\right) \otimes D B,\left(\begin{array}{ll}0 & 0 \\ 1_{F\left(V\left(X_{i+1}\right) \otimes D B\right.} & 0\end{array}\right): F\left(V\left(X_{i+1}\right)\right)\right.$ $\left.\otimes D B \oplus F\left(V\left(X_{i}\right)\right) \otimes D B \otimes D B \rightarrow F\left(V\left(X_{i+2}\right)\right) \oplus F\left(V\left(X_{i+1}\right)\right) \otimes D B\right\}$. Then it is not hard to see that $\mathcal{A}(X)$ and $\mathscr{B}(X)$ become $S_{n}$-modules. We shall define the map $u(X)$ by the following:

$$
\begin{aligned}
& u(X)_{i}=\left(\begin{array}{c}
F\left(\alpha_{X_{i+1}}-\phi_{i}\right) \cdot \eta_{X_{i} \otimes D T} \\
\left(\varepsilon_{V(X)} \otimes D T\right)^{-1} \cdot \alpha_{X_{i}} \otimes D T
\end{array}\right): \\
& \mathcal{A}(X)_{i}=X_{i} \otimes D T \longrightarrow F\left(V\left(X_{i+1}\right)\right) \oplus F\left(V\left(X_{i}\right)\right) \otimes D B=\mathscr{B}(X)_{i} .
\end{aligned}
$$

To see that the above map $u(X)$ is an $S_{n}$-morphism, it is enough to show the commutativity of the following diagram: 


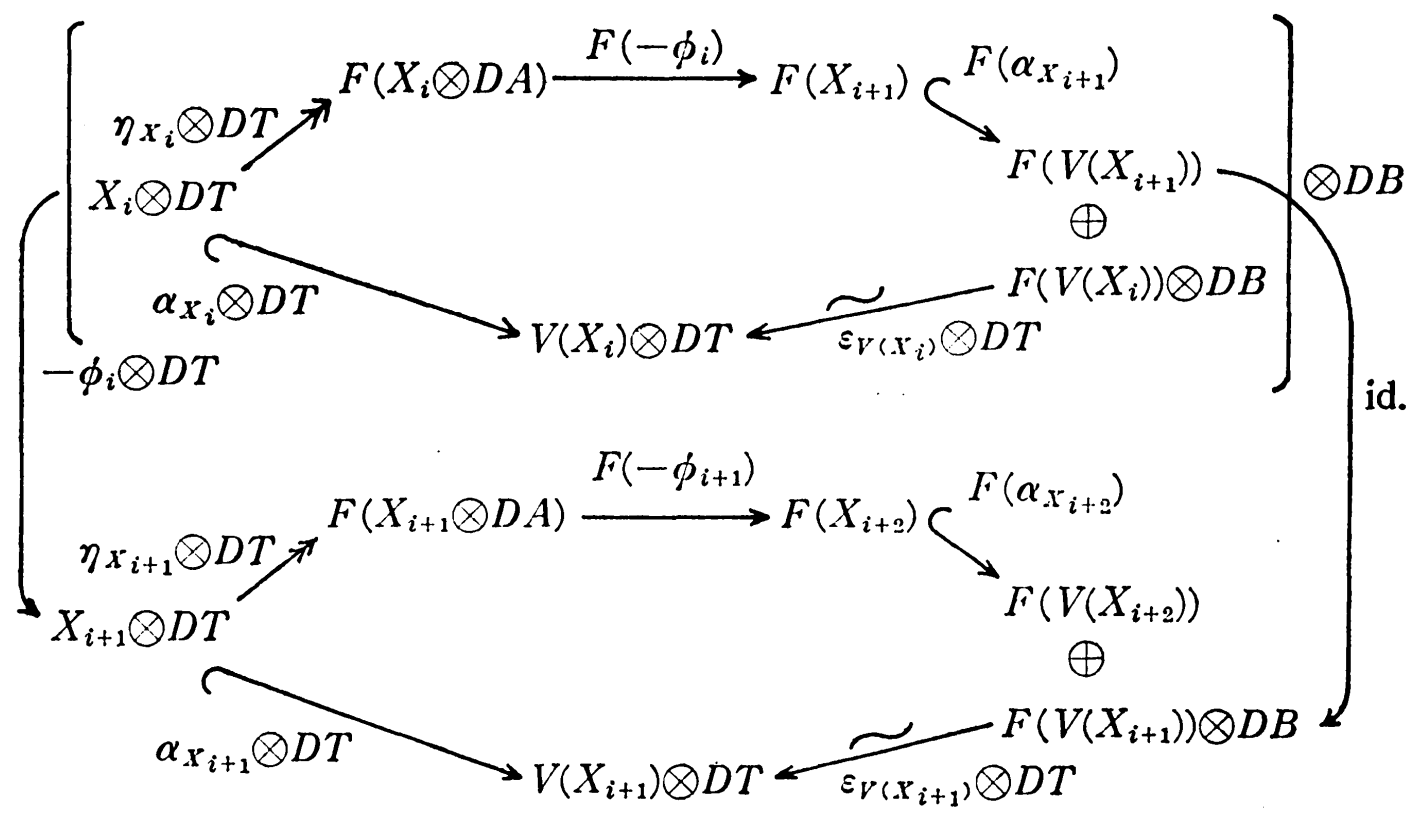

LemMA 2.2. The above diagram is commutative.

Proof. From the naturality of the $\varepsilon$ and $\eta$, we have the following equalities :

$$
\begin{aligned}
& \varepsilon_{V\left(X_{i+1}\right)} \otimes D T \cdot F\left(\alpha_{X_{i+1}}-\phi_{i}\right) \otimes D B \cdot \eta_{X_{i} \otimes D T} \otimes D B \\
& =\left(\alpha_{X_{i+1}} \cdot-\phi_{i}\right) \otimes D T \cdot \varepsilon_{X_{i} \otimes D A} \otimes D T \cdot \eta_{X_{i} \otimes D T} \otimes D B \\
& =\left(\alpha_{X_{i+1}} \cdot-\phi_{i}\right) \otimes D T \cdot\left(\varepsilon_{X_{i} \otimes D T \otimes T} \cdot \eta_{X_{i} \otimes D T} \otimes T\right) \otimes D T \\
& =\left(\alpha_{X_{i+1}} \cdot-\phi_{i}\right) \otimes D T \cdot 1_{X_{i} \otimes D T \otimes D B} \\
& =\alpha_{X_{i+1}} \otimes D T \cdot\left(-\phi_{i} \otimes D T\right), \\
& F\left(\alpha_{X_{i+2}} \cdot-\phi_{i+1}\right) \cdot \eta_{X_{i+1} \otimes D T} \cdot-\phi_{i} \otimes D T \\
& =F\left(\alpha_{X_{i+2}} \cdot-\phi_{i+1}\right) \cdot F\left(-\phi_{i} \otimes D A\right) \cdot \eta_{X_{i} \otimes D A D D T} \\
& =F\left(\alpha_{X_{i+2}}\right) \cdot F\left(\phi_{i+1} \cdot \phi_{i} \otimes D A\right) \cdot \eta_{X_{i} \otimes D A D D T}=0 .
\end{aligned}
$$

The desired commutativity follows from the above equalities.

Since $\alpha_{X_{i}} \otimes D T$ is an injection and $\varepsilon_{V\left(X_{i}\right)} \otimes D T$ is a bijection, $u(X)_{i}$ is also an injection for each $i$. Therefore, $u(X)$ is an $S_{n}$-monomorphism. Thus we can define the $S_{n}$-module $\mathcal{S}_{n}(X)$ as the cokernel Cok $u(X)$ of this $S_{n}$-monomorphism $u(X)$.

From the definition of $\mathcal{S}_{n}$, the following lemma is easily checked.

LEMMA 2.3. For any projective $R_{n}$-module $P$, the $S$-module $\mathcal{S}_{n}(P)$ is also 
projective.

The remaining part of this section is devoted to the proof of the following proposition.

PROPOSITION 2.4. The correspondence $\mathcal{S}_{n}$ can be seen as a stable functor from $\underline{\bmod }-R_{n}$ to $\underline{\bmod }-S_{n}$.

It is necessary to define the $S_{n}$-morphism $\mathcal{S}_{n}(f)$ for any $R_{n}$-morphism $f=$ $\left\{f_{i}\right\}: X=\left\{X_{i}, \phi_{i}\right\} \rightarrow\left\{X_{i}^{\prime}, \phi_{i}^{\prime}\right\}=X^{\prime}$, at first. In order to define such a morphism, it is sufficient to define $S_{n}$-morphisms $\mathcal{A}(f): \mathcal{A}(X) \rightarrow \mathcal{A}\left(X^{\prime}\right)$ and $\mathscr{B}(f): \mathscr{B}(X) \rightarrow \mathscr{B}\left(X^{\prime}\right)$ such that $u\left(X^{\prime}\right) \cdot \mathcal{A}(f)=\mathscr{B}(f) \cdot u(X)$.

Let us put $\mathcal{A}(f)$ and $\mathscr{B}(f)$ as follows:

$$
\begin{aligned}
& \mathcal{A}(f)_{i}=f_{i} \otimes D T: \mathcal{A}(X)_{i}=X_{i} \otimes D T \longrightarrow X_{i}^{\prime} \otimes D T=\mathcal{A}\left(X^{\prime}\right)_{i}, \\
& \mathscr{B}(f)_{i}=\left(\begin{array}{cc}
F\left(f_{i+1}^{*}\right) & \\
0 & F\left(f_{i}^{*}\right) \otimes D B
\end{array}\right): \\
& \mathscr{B}(X)_{i}=F\left(V\left(X_{i+1}\right)\right) \oplus F\left(V\left(X_{i}\right)\right) \otimes D B \longrightarrow F\left(V\left(X_{i+1}^{\prime}\right)\right) \oplus F\left(V\left(X_{i}^{\prime}\right)\right) \otimes D B=\mathscr{B}\left(X^{\prime}\right)_{i},
\end{aligned}
$$

where $f_{i}^{*}$ is defined by the following commutative diagram

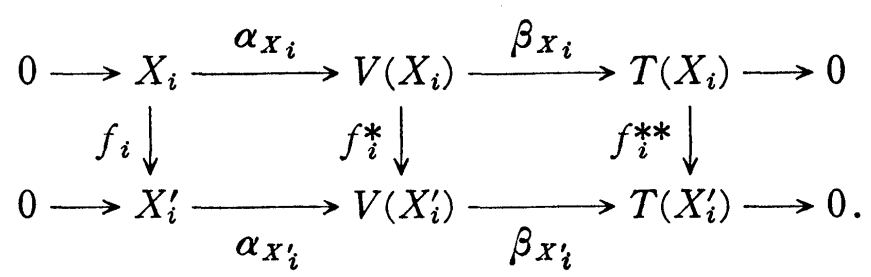

The fact that $\mathcal{A}(f)$ and $\mathscr{B}(f)$ are $S_{n}$-morphisms is clear.

LEMMA 2.5. The above morphisms $\mathcal{A}(f)$ and $\mathscr{B}(f)$ satisfy $u\left(X^{\prime}\right) \cdot \mathcal{A}(f)=\mathscr{B}(f)$ $\cdot u(X)$.

ProOF. We have to verify the following two equalities:

(a) $\varepsilon_{V\left(X_{i}^{\prime}\right)} \otimes D T \cdot F\left(f_{i}^{*}\right) \otimes D B \cdot\left(\varepsilon_{V\left(x_{i}\right)} \otimes D T\right)^{-1} \cdot \alpha_{X_{i}} \otimes D T=\alpha_{X_{i}^{\prime}} \otimes D T \cdot f_{i} \otimes D T$ and

(b) $F\left(f_{i+1}^{*} \cdot \alpha_{X_{i+1}} \cdot-\phi_{i}\right) \cdot \eta_{X_{i} \otimes D T}=F\left(\alpha_{X_{i+1}^{\prime}} \cdot-\phi_{i}^{\prime}\right) \cdot \eta_{X_{i \otimes D T}^{\prime}} \cdot f_{i} \otimes D T$.

The above two equalities (a) and (b) follow from the naturality of $\varepsilon$ and $\eta$ and the following three equalities : $f_{i+1} \cdot \phi_{i}=\phi_{i}^{\prime} \cdot f_{i} \otimes D A, f_{i}^{*} \cdot \alpha_{X_{i}}=\alpha_{X_{i}^{\prime}} \cdot f_{i}$ and $f_{i+1}^{*} \cdot \alpha_{X_{i+1}}$ $=\alpha_{X_{i}^{\prime}} \cdot f_{i+1}$

Therefore we have defined the $S_{n}$-morphism $\mathcal{S}(f)$ by the following commuta- 
tive diagram:

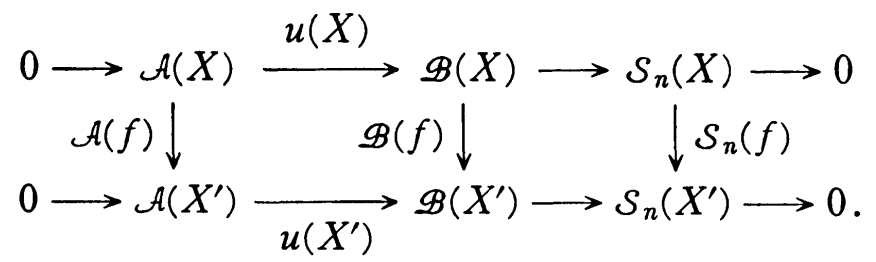

By the definition, $\mathcal{A}(f)$ is uniquely determined by $f$ but $\mathscr{B}(f)$ is not and so $\mathcal{S}_{n}(f)$ is not uniquely determined by $f$. However, in the stable category mod- $S_{n}$, we can prove the singleness of the morphism $\mathcal{S}_{n}(f)$. To show this fact, we shall prove that $\mathcal{S}_{n}(f)$ factors through projective $S_{n}$-modules if $f=0$.

Since $f_{i}=0$, there is a morphism $\delta_{i}: T\left(X_{i}\right) \rightarrow V\left(X_{i}^{\prime}\right)$ and $f_{i}^{*}=\delta_{i} \cdot \beta_{X_{i}}$. Let $\mathscr{Q}(X)$ be a projective $S_{n}$-module defined by

and

$$
\mathscr{P}(X)_{i}=F\left(T\left(X_{i+1}\right)\right) \oplus F\left(T\left(X_{i}\right)\right) \otimes D B
$$

$$
\begin{aligned}
& \left(\mathscr{P}(X)_{i} \otimes D B \rightarrow \mathscr{P}(X)_{i+1}\right)=\left(\begin{array}{ll}
0 & 0 \\
1_{F\left(T\left(X_{i+1}\right)\right) \otimes D B} & 0
\end{array}\right): \\
& F\left(T\left(X_{i+1}\right)\right) \otimes D B \oplus F\left(T\left(X_{i}\right)\right) \otimes D B \otimes D B \longrightarrow F\left(T\left(X_{i+2}\right)\right) \oplus F\left(T\left(X_{i+1}\right)\right) \otimes D B .
\end{aligned}
$$

It is possible to define $S_{n}$-morphisms $\beta(X)$ from $\mathscr{B}(X)$ to $\mathscr{P}(X)$ and $\Delta$ from $\mathscr{Q}(X)$ to $\mathcal{B}\left(X^{\prime}\right)$ so that $\mathscr{B}(f)=\Delta \cdot \beta(X)$ by putting:

$$
\beta(X)_{i}=F\left(\beta_{X_{i+1}}\right) \oplus F\left(\beta_{X_{i}}\right) \otimes D B:
$$$$
F\left(V\left(X_{i+1}\right)\right) \oplus F\left(V\left(X_{i}\right)\right) \otimes D B \longrightarrow F\left(T\left(X_{i+1}\right)\right) \oplus F\left(T\left(X_{i}\right)\right) \otimes D B
$$

and

$$
\begin{aligned}
& \Delta_{i}=F\left(\delta_{i+1}\right) \oplus F\left(\delta_{i}\right) \otimes D B: \\
& F\left(T\left(X_{i+1}\right)\right) \oplus F\left(T\left(X_{i}\right)\right) \otimes D B \longrightarrow F\left(V\left(X_{i+1}^{\prime}\right)\right) \oplus F\left(V\left(X_{i}^{\prime}\right)\right) \otimes D B .
\end{aligned}
$$

It is easy to see that $\beta(X) \cdot u(X)=0$ and $\mathcal{S}_{n}(f)$ factors through projective $S_{n}$ module $\mathscr{P}(X)$ :

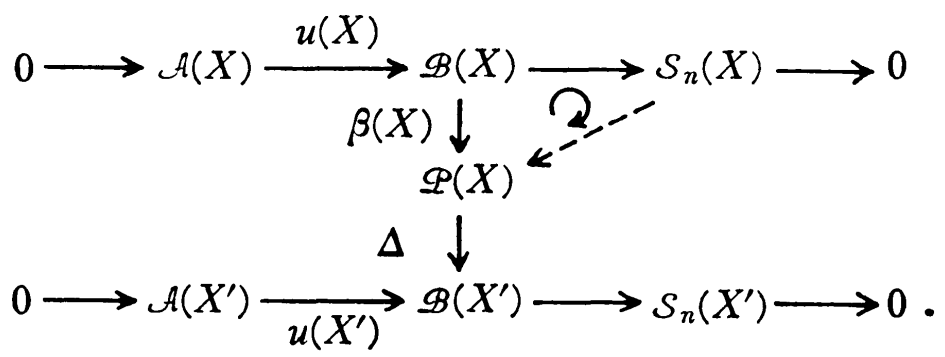

Therefore, we have defined the functor nod- $R_{n} \rightarrow \bmod -S_{n}$ and this functor induces the desired stable functor ${ }^{-} S_{n}: \bmod -R_{n} \rightarrow \underline{\bmod }-S_{n}$ by Lemma 2.3. This completes the proof of Proposition 2.4. 
From the definition of the functors $\Phi_{n}, \Phi_{m, n}$ and $\mathcal{S}_{n}$, the commutativity of the diagram in Introduction is now obvious.

\section{The functor $Q_{n}: \underline{\bmod }-S_{n} \rightarrow \underline{\bmod }-R_{n}$}

The functor $Q_{n}$ has defined as the composite $\underline{\bmod }-S_{n} \stackrel{D}{\longrightarrow} S_{n}$ - $\underline{\bmod } \stackrel{\mathcal{S}_{n}}{\longrightarrow} R_{n}$ - $\underline{\bmod }$ $D$ mod- $R_{n}$. In this section, we shall show the construction of this functor in an explicit way, for the later use.

In the definition of the functor $\mathcal{S}_{n}$, we expressed $R_{n^{-}}$and $S_{n}$-modules as the tensor forms: $\left\{X_{i}, \phi_{i}: X_{i} \otimes D A \rightarrow X_{i+1}\right\}$ and $\left\{Y_{i}, \psi_{i}: Y_{i} \otimes D B \rightarrow Y_{i+1}\right\}$. But for the definition of the functor $Q_{n}$, it is convenient to express the modules as the hom-forms : $\left\{X_{i}, \bar{\phi}_{i}: X_{i} \rightarrow \operatorname{Hom}_{A}\left(D A, X_{i+1}\right)\right\}$ and $\left\{Y_{i}, \bar{\phi}_{i}: Y_{i} \rightarrow \operatorname{Hom}_{B}\left(D B, Y_{i+1}\right)\right\}$, where $\bar{\phi}_{i}$ (resp. $\bar{\phi}_{i}$ ) is the adjoint of $\phi_{i}$ (resp. $\phi_{i}$ ) which corresponds to $\phi_{i}$ (rep. $\left.\phi_{i}\right)$ by the canonical isomorphism $\operatorname{Hom}_{A}\left(X_{i} \otimes_{A} D A, X_{i+1}\right) \cong \operatorname{Hom}_{A}\left(X_{i}, \operatorname{Hom}_{A}(D A\right.$, $\left.X_{i+1}\right)$ ) (resp. $\operatorname{Hom}_{B}\left(Y_{i} \otimes_{B} D B, Y_{i+1}\right) \cong \operatorname{Hom}_{B}\left(Y_{i}, \operatorname{Hom}_{B}\left(D B, Y_{i+1}\right)\right)$ ).

In the following we shall sometimes abbroviate Hom(?, ?) by [?, ?].

For an $S_{n}$-module $Y=\left\{Y_{i}, \bar{\phi}_{i}\right\}$, let us put

$$
\begin{aligned}
\mathcal{C}(Y) & =\left\{\left[D T, Y_{i}\right],\left[D T,-\bar{\psi}_{i}\right]:\left[D T, Y_{i}\right] \longrightarrow\left[D T,\left[D B, Y_{i+1}\right]\right]\right. \\
& \left.=\left[D A,\left[D T, Y_{i+1}\right]\right]\right\}
\end{aligned}
$$

and

$$
\begin{gathered}
\mathscr{D}(Y)=\left\{\left[D A, G\left(U\left(Y_{i}\right)\right)\right] \oplus G\left(U\left(Y_{i-1}\right)\right),\left(\begin{array}{cc}
0 & 0 \\
{\left[D A, G\left(U\left(Y_{i}\right)\right)\right]} & 0
\end{array}\right):\right. \\
{\left[D A, G\left(U\left(Y_{i}\right)\right)\right] \oplus G\left(U\left(Y_{i-1}\right)\right) \longrightarrow\left[D A,\left[D A, G\left(U\left(Y_{i+1}\right)\right)\right]\right]} \\
\left.\bigoplus\left[D A, G\left(U\left(Y_{i}\right)\right)\right]\right\}
\end{gathered}
$$

and define the map $p(Y): \mathscr{D}(Y) \rightarrow \mathcal{C}(Y)$ as follows:

$$
\begin{aligned}
& p(Y)_{i}=\left(\left[D T, \gamma_{Y_{i}} \cdot\left(\eta_{U\left(Y_{i}\right)}\right)^{-1}, \varepsilon_{\left[D T, Y_{i}\right]} \cdot G\left(-\bar{\psi}_{i} \cdot \gamma_{Y_{i-1}}\right):\right.\right. \\
& \mathscr{D}(Y)_{i}=\left[D A, G\left(U\left(Y_{i}\right)\right)\right] \oplus G\left(U\left(Y_{i-1}\right)\right) \longrightarrow\left[D T, Y_{i}\right]=\mathcal{C}(Y)_{i} .
\end{aligned}
$$

Then $\mathcal{C}(Y)$ and $\mathscr{D}(Y)$ become $R_{n}$-modules and $p(Y)$ is an $R_{n}$-morphism. The mhdule $Q_{n}(Y)$ coincides with the kernel $\operatorname{Ker} p(Y)$ of the above morphism $p(Y)$.

For an $S_{n}$-morphism $g=\left\{g_{i}: Y_{i} \rightarrow Y_{i}^{\prime}\right\}: Y=\left\{Y_{i}, \bar{\phi}_{i}\right\} \rightarrow Y^{\prime}=\left\{Y_{i}^{\prime}, \bar{\psi}_{i}^{\prime}\right\}$, we put $\mathcal{C}(g): \mathcal{C}(Y) \rightarrow \mathcal{C}\left(Y^{\prime}\right)$ and $\mathscr{D}(g): \mathscr{D}(Y) \rightarrow \mathscr{D}\left(Y^{\prime}\right)$ as follows :

$$
\begin{aligned}
& \mathcal{C}(g)_{i}=\left[D T, g_{i}\right]:\left[D T, Y_{i}\right] \longrightarrow\left[D T, Y_{i}^{\prime}\right], \\
& \mathscr{D}(g)_{i}=\left(\begin{array}{cc}
{\left[D A, G\left(g_{i}^{*}\right)\right]} & 0 \\
0 & G\left(g_{i-1}^{*}\right)
\end{array}\right): \\
& {\left[D A, G\left(U\left(Y_{i}\right)\right)\right] \oplus G\left(U\left(Y_{i-1}\right)\right) \longrightarrow\left[D A, G\left(U\left(Y_{i}^{\prime}\right)\right)\right] \oplus G\left(U\left(Y_{i-1}^{\prime}\right)\right),}
\end{aligned}
$$


where $g_{i}^{*}$ is defined by the following commutative diagram:

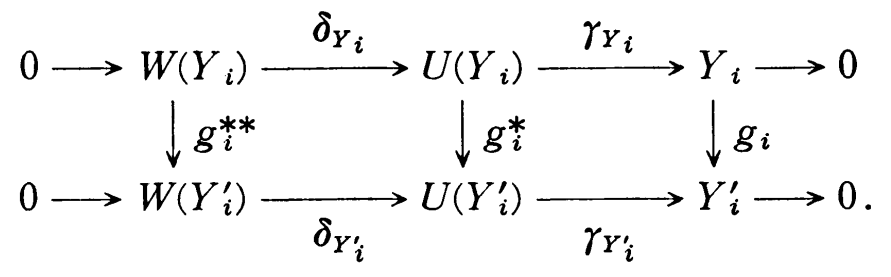

Then $\mathcal{C}(g)$ and $\mathscr{D}(g)$ become $R_{n}$-morphisms and satisfy $\mathcal{C}(g) \cdot p(Y)=p\left(Y^{\prime}\right) \cdot \mathscr{D}(g)$. The $R_{n}$-morphism $Q_{n}(g): Q_{n}(Y) \rightarrow Q_{n}\left(Y^{\prime}\right)$ is defined by the following commutative diagram:

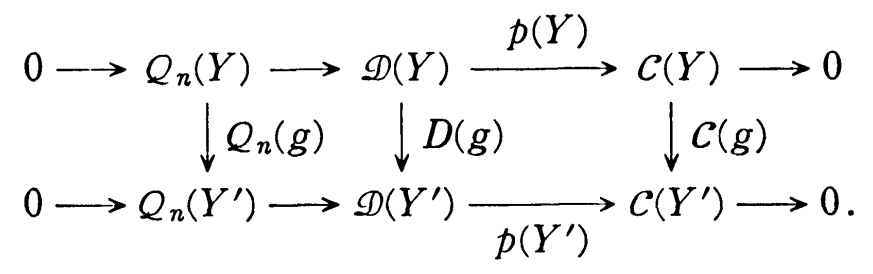

Similarly to the functor $\mathcal{S}_{n}, Q_{n}$ can be considered as a functor mod- $S_{n} \rightarrow$ $\underline{\bmod }-R_{n}$ and it induces $\underline{\bmod }-S_{n} \rightarrow \underline{\bmod }-R_{n}$.

\section{The proof of the isomorphism $Q_{n} \cdot \mathcal{S}_{n} \cong 1_{\underline{m o d} \cdot R_{n}}$}

We begin with the survey of the torsion-free resolution of the component of $\mathcal{S}_{n}(X)$, in order to investigate the module $Q_{n} \mathcal{S}_{n}(X)$.

Let us denote the morphism cok $u(X)$ by $\theta(X)$ :

$$
\theta(X)_{i}=\left(x_{i}, y_{i}\right): F\left(V\left(X_{i+1}\right)\right) \oplus F\left(V\left(X_{i}\right)\right) \otimes D B \longrightarrow \mathcal{S}_{n}(X)_{i} .
$$

Let $P_{0}^{i} \stackrel{p_{0}^{i}}{\longrightarrow} V\left(X_{i}\right) \rightarrow 0$ be the projective cover, then we have the following commutative diagram with exact rows:

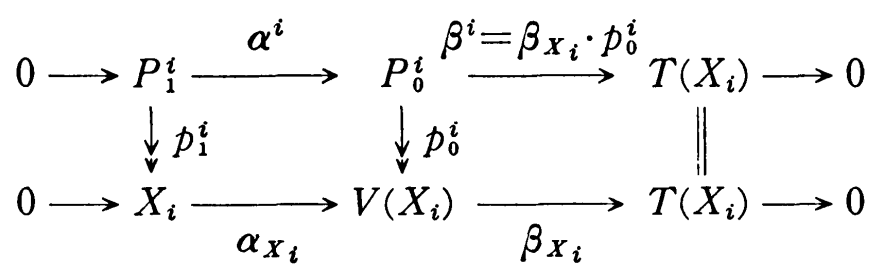

Since proj. dim. $T_{A} \leqq 1, P_{1}^{i}$ has to be projective. Applying $\left(? \otimes_{A} D T\right)$ to the above diagram, we have the following commutative diagram:

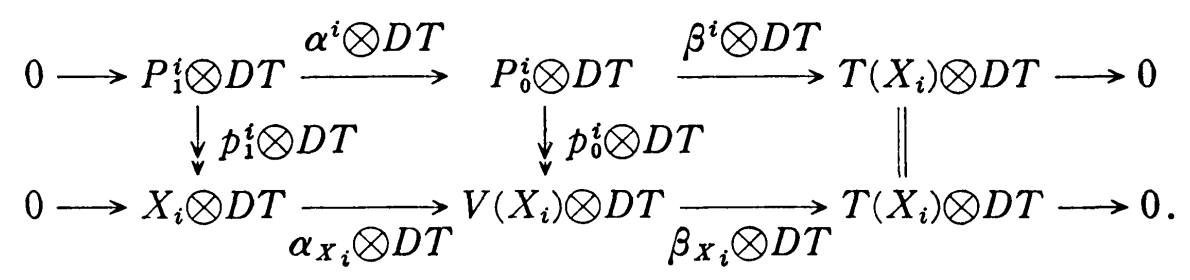


Here we used the fact that $\operatorname{Tor}_{1}^{A}\left(T\left(X_{i}\right), D T\right) \cong D \operatorname{Ext}_{A}^{1}\left(T\left(X_{i}\right), T\right)=0$. Hence we know $\operatorname{Ker}\left(p_{1}^{i} \otimes D T\right)_{B} \cong \operatorname{Ker}\left(p_{0}^{i} \otimes D T\right)_{B}$ by the Snake Lemma.

Consider the following diagram of $B$-modules:

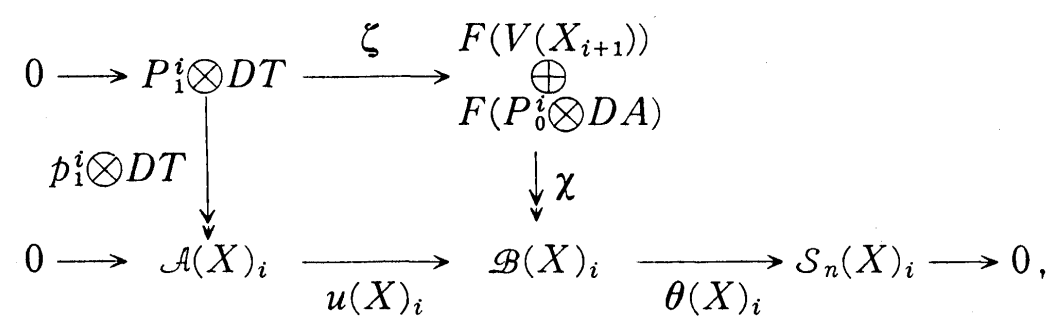

where $\zeta$ and $\chi$ are defined as follows :

$$
\begin{aligned}
& \zeta=\left(\begin{array}{c}
F\left(\alpha_{X_{i}} \cdot-\phi_{i}\right) \cdot \eta_{X_{i} \otimes D T} \cdot p_{1}^{i} \otimes D T \\
\left(\eta_{P_{0}^{i} \otimes D T}\right)^{-1} \cdot \alpha^{i} \otimes D T
\end{array}\right), \\
& \chi=\left(\begin{array}{cc}
1_{F\left(V\left(X_{i+1}\right)\right)} & 0 \\
0 & \left(\varepsilon_{V\left(X_{i}\right)} \otimes D T\right)^{-1} \cdot p_{0}^{i} \otimes D T \cdot\left(\eta_{P_{0}^{i} \otimes D T}\right)^{-1}
\end{array}\right) .
\end{aligned}
$$

From the fact that $\operatorname{Ker}\left(p_{0}^{i} \otimes D T\right)_{B} \cong \operatorname{Ker}\left(p_{1}^{i} \otimes D T\right)_{B}$, it follows that $\operatorname{Ker} \chi \cong \operatorname{Ker}$ $\left(\left(\varepsilon_{V\left(X_{i}\right)} \otimes D T\right)^{-1} \cdot p_{0}^{i} \otimes D T \cdot\left(\eta_{P_{0}^{i} \otimes D T}\right)^{-1}\right) \cong \operatorname{Ker}\left(p_{0}^{i} \otimes D T\right) \cong \operatorname{Ker}\left(p_{1}^{i} \otimes D T\right)$. Therefore we have $\operatorname{Cok} \zeta \cong \mathcal{S}_{n}(X)_{i}$ and we have a torsion-free resolution of $\mathcal{S}_{n}(X)_{i}$.

LEMMA 4.1. The following exact sequence is a torsion-free resolution of $\mathcal{S}_{n}(X)_{i}$ :

$$
0 \longrightarrow P_{1}^{i} \otimes D T \stackrel{\zeta}{\longrightarrow} F\left(V\left(X_{i+1}\right)\right) \oplus F\left(P_{0}^{i} \otimes D A\right) \longrightarrow \mathcal{S}_{n}(X)_{i} \longrightarrow 0 .
$$

It is clear that $P_{1}^{i} \otimes D T \in \operatorname{add}\left(D T_{B}\right)$ and $F\left(F\left(X_{i+1}\right)\right) \oplus F\left(P_{0}^{i} \otimes D A\right) \in \mathscr{G}$. We shall denote coker $\zeta$ by $\hat{\theta}_{i}=\left(x_{i}, \hat{y}_{i}\right)$.

To define the modules $\mathcal{S}_{n}(X)$ and $Q_{n}(Y)$, we have used the minimal torsion and torsion-free resolutions. But by the remark on torsion and torsion-free resolutions in section one, we may use any such resolutions since we consider modules in the stable categories.

Now, using the torsion-free resolutions given by Lemma 4.1, let us calculate the module $Q_{n} \mathcal{S}_{n}(X)$.

The routine verification shows the following lemma.

LEMMA 4.2. The map $p\left(\mathcal{S}_{n}(X)\right)$ is expressed as follows:

(a) $\quad \mathcal{C S}_{n}(X)_{i}=\left[D T, \mathcal{S}_{n}(X)_{i}\right]$,

(b) $\mathscr{D S} \mathcal{S}_{n}(X)_{i}=\left[D A, G F\left(V\left(X_{i+1}\right)\right)\right] \oplus\left[D A, G F\left(P_{0}^{i} \otimes D A\right)\right]$

$$
\oplus G F\left(V\left(X_{i}\right)\right) \oplus G F\left(P_{0}^{i-1} \otimes D A\right)
$$


(c) $p\left(\mathcal{S}_{n}(X)\right)_{i}=\left(\left[D T, x_{i} \cdot\left(\eta_{F\left(V\left(x_{i+1}\right)\right.}\right)\right)^{-1}\right],\left[D T, \hat{y}_{i} \cdot\left(\eta_{F\left(P_{0}^{i} \otimes D A\right.}\right)^{-1}\right]$,

$$
\left.\varepsilon_{\left[D T, S_{n}(X)_{i}\right]} \cdot F\left(-\bar{\psi}_{i-1} \cdot x_{i-1}\right), \varepsilon_{\left[D T, S_{n}(X)_{i}\right]} \cdot F\left(-\bar{\psi}_{i-1} \cdot \hat{y}_{i-1}\right)\right),
$$

where we identify $[D A, ?]$ (resp. $[D B, ?])$ with $[D T,[T, ?]]$ (resp. $[T,[D T, ?]])$ and $\bar{\psi}_{i}: \mathcal{S}_{n}(X)_{i} \rightarrow\left[D B, \mathcal{S}_{n}(X)_{i+1}\right]$ denotes the $i$-th structure map of the $S_{n}$-module $\mathcal{S}_{n}(X)$ in the hom-form.

The remaining part of this section is devoted to prove that $\operatorname{Ker} p\left(\mathcal{S}_{n}(X)\right)$ is isomorphic to $X$ as an object in the stable category mod- $R_{n}$. In fact, we shall show $\operatorname{Ker} p\left(\mathcal{S}_{n}(X)\right) \cong X \oplus P$ for the projective (= injective) $R_{n}$-module $P$ defined as follows :

$$
P=\left\{P_{0}^{i} \oplus P_{0}^{i}{ }^{1} \otimes D A,\left(\begin{array}{cc}
0 & 0 \\
1_{P_{0}^{i}} \otimes_{D A} & 0
\end{array}\right)\right\} .
$$

LEMMA 4.3. $\left|\operatorname{Ker} p\left(\mathcal{S}_{n}(X)\right)\right|=|X|+|P|$, where $|*|$ denotes the K-composition length of a module *.

Proof. By Lemma 4.1, we have

$$
\begin{aligned}
\left|\operatorname{Ker} p\left(\mathcal{S}_{n}(X)\right)_{i}\right|= & \left|\left[D A, V\left(X_{i+1}\right)\right]\right|+\left|P_{0}^{i}\right|+\left|V\left(X_{i}\right)\right| \\
& +\left|P_{0}^{i-1} \otimes D A\right|-\left|\left[D T, \mathcal{S}_{n}(X)_{i}\right]\right|,
\end{aligned}
$$

since $\varepsilon_{V}: F G(V) \cong V$ for a torsion $A$-module $V$ and $\eta_{U}: U \cong G F(U)$ for a torsionfree $B$-module $U$ by Brenner-Butler's theorem. On the other hand, from the exact sequence

$$
0 \longrightarrow P_{1}^{i} \otimes D T \longrightarrow F\left(V\left(X_{i+1}\right)\right) \oplus F\left(P_{0}^{i} \otimes D A\right) \longrightarrow \mathcal{S}_{n}(X)_{i} \longrightarrow 0
$$

we have the exact sequence

$$
\begin{aligned}
0 \longrightarrow\left[D T, P_{1}^{i} \otimes D T\right] & \longrightarrow\left[D T, F\left(V\left(X_{i+1}\right)\right)\right] \oplus\left[D T, F\left(P_{0}^{i} \otimes D A\right)\right] \\
& \longrightarrow\left[D T, \mathcal{S}_{n}(X)_{i}\right] \longrightarrow 0
\end{aligned}
$$

and $\left[D T, F\left(P_{0}^{i} \otimes D A\right)\right] \cong\left[D A, P_{0}^{i} \otimes D A\right] \cong P_{0}^{i},\left[D T, P_{1}^{i} \otimes D T\right] \cong\left[D T, D\left[P_{1}^{i}, T\right]\right] \cong$ $\left[\left[P_{1}^{i}{ }^{\prime} T\right], T\right] \cong P_{1}^{i}$, as well. Therefore, it follows $\left|\left[D T, \mathcal{S}_{n}(X)_{i}\right]\right|=\left|\left[D A, V\left(X_{i+1}\right)\right]\right|$ $+\left|P_{0}^{i}\right|-\left|P_{1}^{i}\right|$. Hence we have $\left|\operatorname{Ker} p\left(\mathcal{S}_{n}(X)\right)_{i}\right|=\left|V\left(X_{i}\right)\right|+\left|P_{0}^{i-1} \otimes D A\right|+\left|P_{1}^{i}\right|$. Further, from the exact commutative diagram :

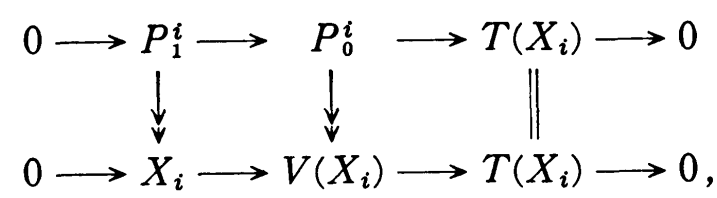

we know $\left|P_{0}^{i}\right|-\left|P_{1}^{i}\right|=\left|T\left(X_{i}\right)\right|=\left|V\left(X_{i}\right)\right|-\left|X_{i}\right|$, i.e., $\left|V\left(X_{i}\right)\right|+\left|P_{1}^{i}\right|=\left|P_{0}^{i}\right|+\left|X_{i}\right|$. 
Finally, we have $\left|\operatorname{Ker} p\left(\mathcal{S}_{n}(X)\right)_{i}\right|=\left|P_{0}^{i-1} \otimes D A\right|+\left|P_{0}^{i}\right|+\left|X_{i}\right|=\left|X_{i}\right|+\left|P_{i}\right|$ and this means that $\left|\operatorname{Ker} p\left(\mathcal{S}_{n}(X)\right)\right|=|X|+|P|$ as desired.

By the above lemma, in order to prove the isomorphism $\operatorname{Ker} p\left(\mathcal{S}_{n}(X)\right) \cong X \oplus P$, it suffices to show the existence of an $R_{n}$-monomorphism $(e(X), f(X)): X \oplus P \rightarrow$ $\mathscr{D S} \mathcal{S}_{n}(X)$ such that the composition $p\left(\mathcal{S}_{n}(X)\right) \cdot(e(X), f(X))$ is a zero map. To define such morphisms $e(X)$ and $f(X)$, it is necessary to introduce a notation: For a bimodule ${ }_{E_{1}} M_{E_{2}}$ over algebras $E_{1}$ land $E_{2}$, we can always consider the adjoint pair of functors $\operatorname{Hom}_{E_{2}}(M, ?): \bmod -E_{2} \rightarrow \bmod -E_{1}$ and $\left(? \otimes_{E_{1}} M\right): \bmod -E_{1} \rightarrow$ mod- $E_{2}$. We shall denote the unit and counit of this adjunction by $\eta^{M}: 1_{\bmod -E_{1}}$ $\rightarrow \operatorname{Hom}_{E_{2}}\left(M, ? \otimes_{E_{1}} M\right)$ and $\varepsilon^{M}: \operatorname{Hom}_{E_{2}}\left(M\right.$, ?) $\bigotimes_{E_{1}} M \rightarrow 1_{\bmod \cdot E_{2}}$, respectively. Then it is noted that $\eta=\eta^{T}$ and $\varepsilon=\varepsilon^{T}$.

Now let us put $e(X): X \rightarrow \mathscr{D S} \mathcal{S}_{n}(X)$ and $f(X): P \rightarrow \mathscr{D S} \mathcal{S}_{n}(X)$ as follows :

and

$$
e(X)_{i}=\left[\begin{array}{c}
{\left[D A,\left(\varepsilon_{V\left(X_{i+1}\right)}\right)^{-1} \cdot \alpha_{X_{i+1}} \cdot \phi_{i}\right] \cdot \eta_{X_{i}}^{D_{i}}} \\
0 \\
\left(\varepsilon_{V\left(X_{i}\right)}\right)^{-1} \cdot \alpha_{X_{i}} \\
0
\end{array}\right]
$$

$$
f(X)_{i}=\left[\begin{array}{cc}
0 & 0 \\
{\left[D T, \eta_{\left[T, P_{0}^{i} \otimes D A\right]}\right]} & 0 \\
\left(\varepsilon_{V\left(X_{i}\right)}\right)^{-1} \cdot p_{0}^{i} & 0 \\
0 & \left(\varepsilon_{P_{0}^{i-1} \otimes D A}\right)^{-1}
\end{array}\right] .
$$

In the following, we shall show that $e(X)$ and $f(X)$ are $R_{n}$-homomorphisms and $p\left(\mathcal{S}_{n}(X)\right) \cdot e(X)=0=p\left(\mathcal{S}_{n}(X)\right) \cdot f(X)$. To do so, it is necessary to provide the following lemma.

LEMMA 4.4. The following diagrams are commutative for any A-module $X$ and $B$-morphism $g: Z \otimes D B \rightarrow Y$.

(a)

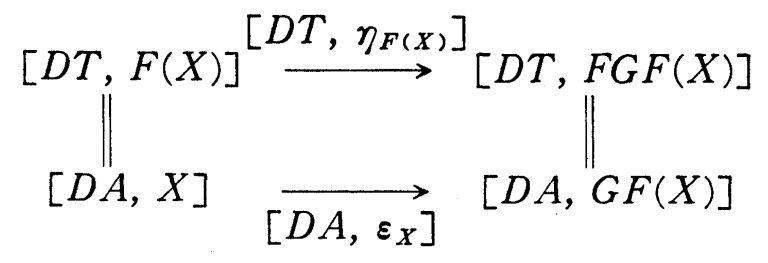

(b)

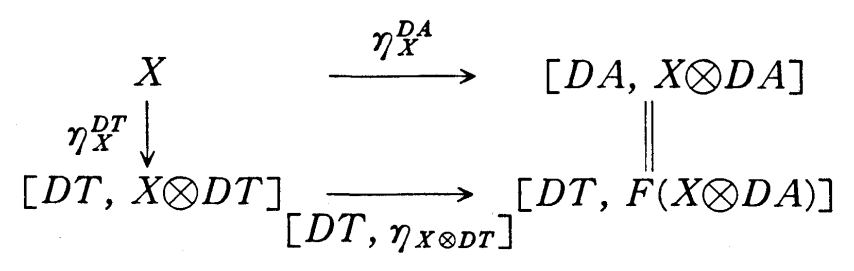


(c)

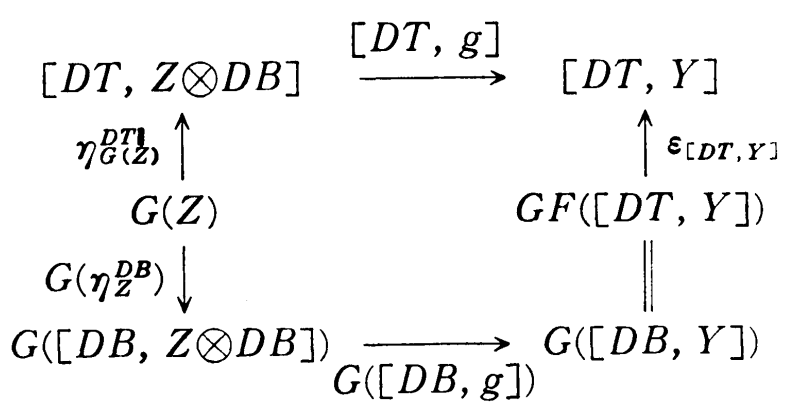

Proof. Rutine verification.

LEMMA 4.5. The map $e(X)$ is an $R_{n}$-morphism.

Proof. At first, we have to verify the equality

$$
\begin{aligned}
& \varepsilon_{G F\left(V\left(X_{i+1}\right)\right.}^{D A} \cdot\left(\left[D A, \varepsilon_{V\left(X_{i+1}\right)}\right] \otimes D A\right)^{-1} \cdot\left[D A, \alpha_{X_{i+1}} \cdot \phi_{i}\right] \otimes D A \cdot \eta_{X_{i}}^{D A} \otimes D A \\
& =\left(\varepsilon_{V\left(X_{i+1}\right)}\right)^{-1} \cdot \alpha_{X_{i+1}} \cdot \phi_{i} .
\end{aligned}
$$

By the naturality of $\varepsilon^{D A}$ and the relation $\varepsilon_{X_{i} \otimes D A}^{D A} \cdot \eta_{X_{i}}^{D A} \otimes D A=1_{X_{i}}$, we have $\alpha_{X_{i+1}}$ - $\phi_{i}=\varepsilon_{V\left(X_{i+1}\right)}^{D A} \cdot\left[D A, \alpha_{X_{i+1}} \cdot \phi_{i}\right] \otimes D A \cdot \eta_{X_{i}}^{D A} \otimes D A$. Hence it is sufficient to show $\left(\varepsilon_{V\left(X_{i+1}\right)}\right)^{-1} \cdot \varepsilon_{V\left(X_{i+1}\right)}^{D A}=\varepsilon_{G F\left(V\left(X_{i+1}\right)\right)}^{D A} \cdot\left(\left[D A, \varepsilon_{V\left(X_{i+1}\right)}\right] \otimes D A\right)^{-1}$, i.e., $\varepsilon_{V\left(X_{i+1}\right)} \cdot \varepsilon_{G F\left(V\left(X_{i+1}\right)\right)}^{D A}$ $=\varepsilon_{V\left(X_{i+1}\right)}^{D A} \cdot\left[D A, \varepsilon_{V\left(X_{i+1}\right)}\right] \otimes D A$. But this follows again from the naturality of $\varepsilon^{D A}$.

The another necessary condition $\left(\left[D A, \varepsilon_{V\left(X_{i+2}\right)}\right]\right)^{-1} \cdot\left[D A, \alpha_{X_{i+2}} \cdot \phi_{i+1}\right] \cdot \eta_{X_{i+1}}^{D A}$ $\cdot \phi_{i}=0$ is obvious.

LEMMA 4.6. The map $f(X)$ is an $R_{n}$-morphism, as well.

Proof. We have to verify the equality

$$
\left(\varepsilon_{P_{0}^{i} \otimes D A}\right)^{-1}=\eta_{G F\left(P_{0}^{i} \otimes D A\right.}^{D A} \cdot\left[D T, \eta_{F\left(P_{0}^{i} \otimes D A\right.}\right] \otimes D A \cdot \eta_{P^{i}}^{D A} .
$$

Since $\varepsilon_{P_{0}^{i \otimes D A}}^{D A} \cdot \eta_{P_{0}^{i}}^{D A} \otimes D A=1_{P_{0}^{i} \otimes D A}$ and $\varepsilon^{D A}$ is a natural transformation, we have

$$
\left(\varepsilon_{P_{0}^{i} \otimes D A}\right)^{-1}=\varepsilon_{G F\left(F_{0}^{i} \otimes D A\right.}^{D A} \cdot\left(\left[D A, \varepsilon_{P_{0}^{i} \otimes D A}\right] \otimes D A\right)^{-1} \cdot \eta_{P_{0}^{i}}^{D A} \otimes D A .
$$

Hence we have the desired result since $\left(\left[D A, \varepsilon_{P_{0}^{i} \otimes D A}\right]\right)^{-1}=\left[D T, \eta_{F\left(P_{0}^{i} \otimes D A\right.}\right] \otimes D A$ by Lemma 4.4 (a).

For the proof of $p(X) \cdot(e(X), f(X))=0$, we note that the $i$-th structure map $\phi_{i}: \mathcal{S}_{n}(X)_{i} \otimes D B \rightarrow \mathcal{S}_{n}(X)_{i+1}$ satisfies $\psi_{i} \cdot y_{i} \otimes D B=0$ and $y_{i+1}=\phi_{i} \cdot x_{i} \otimes D B$ and its adjoint $\bar{\psi}_{i}$ is the same with the composition: $\left[D B, \psi_{i}\right] \cdot \eta_{S_{n}(X)_{i}}^{D B}: \mathcal{S}_{n}(X)_{i} \rightarrow$ $\left[D B, \mathcal{S}_{n}(X)_{i} \otimes D B\right] \rightarrow\left[D B, \mathcal{S}_{n}(X)_{i+1}\right]$. Then it is easy to prove the following lemma, by definition.

LEMMA 4.7. The following hold. 
(a) $G\left(\bar{\psi}_{i-1} \cdot x_{i-1}\right)=G\left(\left[D B, y_{i}\right] \cdot \eta_{F\left(V\left(x_{i}\right)\right)}^{D A}\right)$

(b) $G\left(\bar{\psi}_{i-1} \cdot \hat{y}_{i-1}\right)=0$

LEMMA 4.8. $\quad p(X) \cdot e(X)=0$.

Proof. By Lemma 4.7 (a), it is sufficient to prove the commutativity of the following diagram:

$$
\begin{aligned}
& X_{i} \stackrel{\eta_{X_{i}}^{D_{A}} \longrightarrow}{\longrightarrow}\left[D A, X_{i} \otimes D A\right] \stackrel{\left[D A, \varphi_{i}\right]}{\longrightarrow}\left[D A, X_{i+1}\right] \stackrel{\left[D A, \alpha_{X_{i+1}}\right]}{\longrightarrow}\left[D T, F\left(V\left(X_{i+1}\right)\right)\right]
\end{aligned}
$$

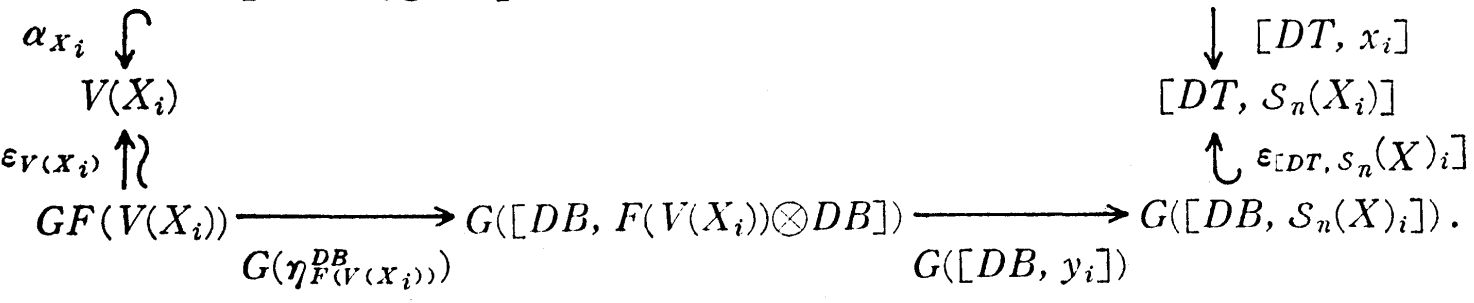

We know $\quad \eta_{X_{i}}^{D A}=\left[D T, \eta_{X_{i} \otimes D T}\right] \cdot \eta_{X_{i}}^{D T}: \quad X_{i} \rightarrow\left[D T, X_{i} \otimes D T\right] \rightarrow\left[D T, F\left(X_{i} \otimes D A\right)\right]=$ $\left[D A, X_{i} \otimes D A\right]$ by Lemma $4.4(\mathrm{~b})$ and $\varepsilon_{\left[D T, s_{n}(X)_{i}\right]} \cdot G\left(\left[D B, y_{i}\right] \cdot \eta_{F^{(V(X)}\left(X_{i}\right)}\right)=\left[D T, y_{i}\right]$ $\cdot \eta_{G F\left(V\left(X_{i}\right)\right)}^{D T}$ be Lemma $4.4(\mathrm{c})$. Further, by the definition of the map $\theta$, it holds that

$$
y_{i} \cdot G\left(\varepsilon_{V\left(X_{i}\right)}\right)^{-1} \cdot \alpha_{X_{i}} \otimes D T=x_{i} \cdot F\left(\alpha_{X_{i+1}} \cdot \phi_{i}\right) \cdot \eta_{X_{i} \otimes D T} .
$$

Hence we have

$$
\begin{aligned}
{[D T} & \left., x_{i}\right] \cdot\left[D A, \alpha_{X_{i+1}} \cdot \phi_{i}\right] \cdot \eta_{X_{i}}^{D A} \\
& =\left[D T, x_{i} \cdot F\left(\alpha_{X_{i+1}} \cdot \phi_{i}\right)\right] \cdot \eta_{X_{i}}^{D A} \\
& =\left[D T, x_{i} \cdot F\left(\alpha_{X_{i+1}} \cdot \phi_{i}\right) \cdot \eta_{X_{i} \otimes D T}\right] \cdot \eta_{X_{i}}^{D T} \\
& =\left[D T, y_{i} \cdot\left(\varepsilon_{V\left(X_{i}\right)} \otimes D T\right)^{-1} \cdot \alpha_{X_{i}} \otimes D T\right] \cdot \eta_{X_{i}}^{D T} \\
& =\left[D T, y_{i} \cdot\left(\varepsilon_{V\left(X_{i}\right)} \otimes D T\right)^{-1}\right] \cdot \eta_{V\left(X_{i}\right)}^{D T} \cdot \alpha_{X_{i}} \\
& =\left[D T, y_{i}\right] \cdot \eta_{G F\left(V\left(X_{i}\right)\right)}^{D T} \cdot\left(\varepsilon_{V\left(X_{i}\right)}\right)^{-1} \cdot \alpha_{X_{i}} \\
& =\varepsilon_{\left[D T, s_{n}(X)_{i}\right]} \cdot G\left(\left[D B, y_{i}\right] \cdot \eta_{F\left(V\left(X_{i}\right)\right)}^{D B}\right) \cdot\left(\varepsilon_{V(X i)}\right)^{-1} \cdot \alpha_{X_{i}}
\end{aligned}
$$

LEMMA 4.9. $p(X) \cdot f(X)=0$.

Proof. By Lemma 4.6 (a), it is enough to prove the commutativity of the diagram : 


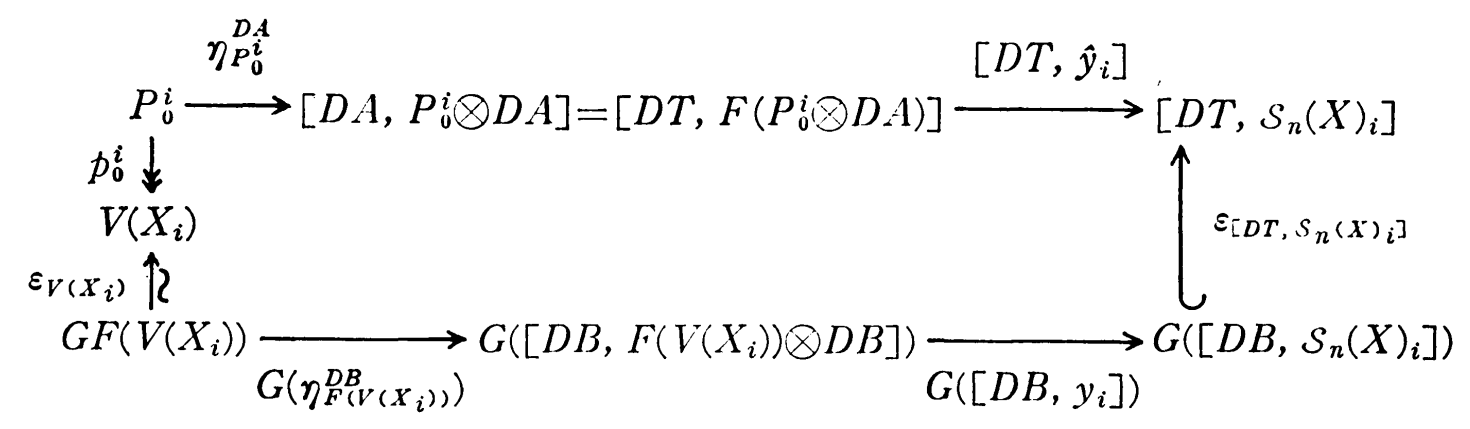

By Lemma 4.4 (b) and (c), we know $\eta_{P_{0}^{i}}^{D A}=\left[D T, \eta_{P_{0}^{i} \otimes D T}^{i}\right] \cdot \eta_{P_{0}^{i}}^{D T}$ and $\eta_{G F\left(V\left(X_{i}\right)\right)}^{D T}=$ $\varepsilon_{\left[D T, F\left(V\left(X_{i}\right)\right) \otimes D B\right]} \cdot G\left(\eta_{F\left(V\left(X_{i}\right)\right)}^{D B}\right.$. Hence we have

$$
\begin{aligned}
{\left[D T, \hat{y}_{i}\right] \cdot \eta_{P_{0}^{i}}^{D A} } & =\left[D T, \hat{y}_{i} \cdot \eta_{P_{0}^{i} \otimes D T}\right] \cdot \eta_{P_{0}^{i}}^{D T} \\
& =\left[D T, y_{i} \cdot\left(\varepsilon_{V\left(X_{i}\right)} \otimes D T\right)^{-1} \cdot p_{0}^{i} \otimes D T\right] \cdot \eta_{P_{0}^{i}}^{D T} \\
& =\left[D T, y_{i} \cdot\left(\varepsilon_{V\left(X_{i}\right)} \otimes D T\right)^{-1}\right] \cdot \eta_{V\left(X_{i}\right)}^{D T} \cdot p_{0}^{i} \\
& =\left[D T, y_{i}\right] \cdot \eta_{G F\left(V\left(X_{i}\right)\right)}^{D T} \cdot\left(\varepsilon_{V\left(X_{i}\right)}\right)^{-1} \cdot p_{0}^{i} \\
& =\left[D T, y_{i}\right] \cdot \varepsilon_{\left[D T, E\left(V\left(X_{i}\right)\right) \otimes D B\right]} \cdot G\left(\eta_{F\left(V\left(X_{i}\right)\right)}^{D B}\right) \cdot\left(\varepsilon_{V\left(X_{i}\right)}\right)^{-1} \cdot p_{0}^{i} \\
& =\varepsilon_{\left[D T, s_{n}(X)_{i}\right]} \cdot G F\left(\left[D T, y_{i}\right]\right) \cdot G\left(\eta_{F\left(V\left(X_{i}\right)\right)}^{D B}\right) \cdot\left(\varepsilon_{V\left(X_{i}\right)}\right)^{-1} \cdot p_{0}^{i} \\
& =\varepsilon_{\left[D T, s_{n}(X)_{i}\right]} \cdot G\left(\left[D B, y_{i}\right] \cdot \eta_{F\left(V\left(X_{i}\right)\right)}^{D B}\right) \cdot\left(\varepsilon_{V\left(X_{i}\right)}\right)^{-1} \cdot p_{0}^{i} .
\end{aligned}
$$

Since $(e(X), f(X))$ is obviously an $R_{n}$-monomorphism, we have proved $Q_{n} \mathcal{S}_{n}(X)$ $\cong X \oplus P$ as $R_{n}$-modules. It is easy to prove that the monomorphism $e(X): X \rightarrow$ $\mathscr{D S}_{n}(X)$ has naturality on $X$. By the duality, we can prove the similar result on $\mathcal{S}_{n} Q_{n}$.

Thus we have

THEOREM 4.10. $Q_{n} \mathcal{S}_{n} \cong 1_{\text {mod. } R_{n}}$ and $\mathcal{S}_{n} Q_{n} \cong 1_{\text {mod. } S_{n}}$, i.e., the stable categories $\underline{\bmod }-R_{n}$ and $\underline{\bmod }-S_{n}$ are always equivalent.

REMARK. D. Happel [15] has proved that $\underline{\bmod }-\hat{A}$ and $\underline{\bmod }-\hat{B}$ are equivalent if gl. $\operatorname{dim} . A<\infty$. And, in the case where $\Phi=\Phi_{1}: \underline{\bmod }-\hat{A} \rightarrow \underline{\bmod }-R$ is dense, the stable functor $\mathcal{S}_{n}$ is induced from $\mathcal{S}_{\infty}$ for each $n \neq \infty$. But, in general, $\Phi$ is not dense even if gl. dim. $A<\infty$, can not be induced from $\mathcal{S}_{\infty}$.

EXAMPLE 4.11. Let $A$ be the bound quiver algebra of

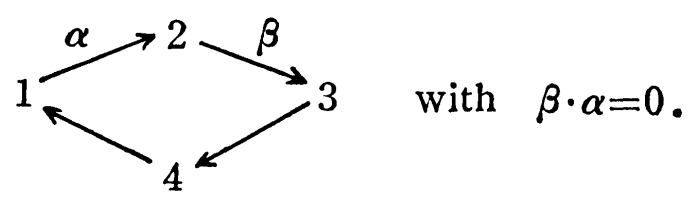


Then $\operatorname{soc}\left(e_{4} A\right) \cong \operatorname{top}\left(e_{4} A \otimes D A\right)$ and there is a non-zero map $f$ from $e_{4} A \otimes D A$ to $e_{4} A$ such that $\operatorname{Im}(f)=\operatorname{soc}\left(e_{4} A\right)$ and $\operatorname{Ker}(f)=\operatorname{rad}\left(e_{4} A \otimes D A\right)$. Hence we can define an indecomposable $R$-module $X=\left(e_{4} A, f\right)$. As is easily seen, for any $R$-module $X^{\prime}, X$ can not be isomorphic to $\Phi\left(X^{\prime}\right)$. Thus $\Phi: \underline{\bmod }-\hat{A} \rightarrow \underline{\bmod }-R$ is not dense, even though g.1 dim. $A=2<\infty$.

\section{Refferences}

[1] Assem, I., Iterated tilted algebras of type $B_{n}$ and $C_{n}$, preprint.

[2] Assem, I. and Happel, D., Generaized tilted algebras of type $A_{n}$, Comm. Algebra 9 (1981), 2101-2125.

[3] Assem, I., Happel D. and Roldan, O., Representation finite trivial extension algebras, preprint.

[4] Assem, I. and Iwanaga, Y., Stable equivalence of representation finite trivial extension, preprint.

[5] Auslander, M., Platzeck, M.I. and Reiten, I., Coxeter functors without diagrams,. Trans. Amer. Math. Soc. 250 (1979), 1-46.

[6] Auslander, M. and Reiten, I., Representation theory of artin algebras III, Comm. Algebra 5 (1975), 239-294.

[7] Auslander, M. and Reiten, I., Representation theory of artin algebras V, Comm. Algebra 5 (1977), 519-554.

[8] Bernstein, I., Gelfand, I. M. and Ponomarev, V.A., Coxeter functor and Gabriel's theorem, Russ. Math. Surveys 28 (1977), 17-32.

[9] Bongartz, K. Tilted algebras, Springer LNM 903 (1981), 16-38.

[10] Brenner, S. and Butler, M.C.R., Generalization of Bernstein-Gelfand-Ponomarev reflection functors, Springer LNM 832 (1980), 103-169.

[11] Dlab, V. and Ringel, C.M., Indecomposable representations of graphs and algebras, Memoirs Amer. Math. Soc. 173 (1976).

[12] Fossum, R.M., Griffith, P.A. and Reiten, I., Trivial extensions of abelian categories, Springer LNM 456 (1975).

[13] Gabriel, P. and Riedtmann, C., Group representations without groups, Comment. Math. Helv. 54 (1979), 240-287.

[14] Gabriel, P., Auslander-Reiten sequences and representation finite algebras, Springer LNM 831 (1980), 1-71.

[15] Happel, D., Triangulated categories and trivial extension algebras, to appear in the proceedings of Carleton Univ., 1984.

[16] Happel, D. and Ringel, C. M., Tilted algebras, Trans. Amer. Math. Soc. 247 (1982), 399-443.

[17] Hoshino, M., Trivial extensions of tilted algebras, Comm. Algeba 10 (1982), 19651999.

[18] Hughes, D. and Waschbüsch, J., Trivial extensions of tilted algebras, Proc. London Math. Soc. 46 (1983), 427-440.

[19] Riedtmann, C., Algebren, Darstellungsköcher, Uberlagerungen und züruck, Comment. Math. Helv. 55 (1980), 199-224.

[20] Tachikawa, H., Representations of trivial extensions of hereditary algebras, Springer LNM 832 (1980), 572-599.

[21] Tachikawa, H., Reflection functors and Auslander-Reiten translations for trivial extensions of hereditary algebras, J. Algebra. 90 (1984), 98-118.

[22] Tachikawa, H., Self-injective algebras and tilting theory, to appear in the proceed- 
ings of Carleton Univ., 1984.

[23] Tachikawa H. and Wakamatsu, T., Extensions of tilting functors and QF-3 algebras, to appean in J. Algebra.

[24] Tachikawa, H. and Wakamatsu, T., Tilting functors and stable equivalences for self-injective algebras, preprint.

[25] Tachikawa, H. and Wakamatsu, T., Applications of reflection functors for selfinjective algebras, to appear in the proceedings of Carleton Univ., 1984.

[26] Wakamatsu, T., Note on trivial extensions of artin algebras, Comm. Algebra 12 (1984), 33-41.

[27] Wakamatsu, T., Partial Coxeter functors and stable equivalences for self-injective algebras, Tsukuba J. Math. 9 (1985), 171-183.

[28] Waschbüsch, J., Universal coverings of self-injective algebras, Springer LNM 903 (1981), 331-349.

[29] Yamagata, K., On algebras whose trivial extensions are of finite representation type, Springer LNM 903 (1981), 364-371.

Institute of Mathematics,

University of Tsukuba

Sakura-mura, Ibaraki,

305, Japan 